\title{
Short communication: Assessment of biomarkers of inflammation in the vaginal discharge of postpartum dairy cows diagnosed with clinical metritis
}

\author{
A. A. Barragan, ${ }^{1 *}$ J. Lakritz, ${ }^{2}$ M. K. Carman, ${ }^{2}$ S. Bas, ${ }^{3}$ E. Hovingh, ${ }^{1}$ and G. M. Schuenemann ${ }^{4}$ \\ ${ }^{1}$ Department of Veterinary and Biomedical Sciences, Pennsylvania State University, University Park 18602 \\ ${ }^{2}$ Department of Veterinary Clinical Sciences, Ohio State University, Columbus 43210 \\ ${ }^{3}$ Phytobiotics Futterzusatzstoffe GmbH Bvd, Villa Maria, Córdoba 5220, Argentina \\ ${ }^{4}$ Department of Veterinary Preventive Medicine, College of Veterinary Medicine, Ohio State University, Columbus 43210
}

\section{ABSTRACT}

Clinical metritis, characterized by the presence of an enlarged uterus and abnormal red-brownish foulsmelling vaginal discharge (VD), is a prevalent condition that causes important economic losses to dairy operations. The accurate diagnosis and treatment of this disease can help decrease its negative effects on the well-being and performance of dairy cows. The objectives of this study were to assess (1) the concentration of haptoglobin (Hp) and neutrophil-derived haptoglobin-matrix metalloproteinase 9 (Hp-MMP 9) in the VD of postpartum cows; and (2) the correlation between Hp and Hp-MMP 9 concentrations in serum and VD. Fiftythree dairy cows from 4 farms in central Pennsylvania were enrolled in this observational study.. Postpartum cows ( \pm 3 DIM) were screened using a Metricheck device to assess VD score (VDS): 1, clear fluid $(\mathrm{n}=4)$; $2,<50 \%$ white purulent fluid $(\mathrm{n}=14) ; 3,>50 \%$ white purulent fluid $(\mathrm{n}=8) ; 4$, red-brownish watery fluid without fetid smell $(\mathrm{n}=22)$; and 5 , fetid red-brownish watery fluid $(\mathrm{n}=5)$. Blood and VD samples were collected for assessment of Hp and Hp-MMP 9 concentrations. Cows with a VDS of 4 or 5 (VDS4/5) had higher serum Hp concentrations than cows with a VDS of 1,2 , or $3(\mathrm{VDS} 1 / 3 ; 93 \pm 187 \mu \mathrm{g} / \mathrm{mL}$ vs. $59 \pm 106 \mu \mathrm{g} / \mathrm{mL}$, respectively). Similarly, cows with VDS4/5 had higher VD Hp concentrations than cows with VDS1/3 (73 \pm $56 \mu \mathrm{g} / \mathrm{mL}$ vs. $17 \pm 16 \mu \mathrm{g} / \mathrm{mL}$, respectively). We found a significant correlation (0.37) between Hp levels in serum and in VD. We found no difference in serum HpMMP 9 between VDS4/5 and VDS1/3 cows. The VD concentrations of Hp-MMP 9 were higher in VDS4/5 cows than in VDS1/3 cows $(7,629 \pm 9,847 \mathrm{ng} / \mathrm{mL}$ vs.

Received October 15, 2018.

Accepted April 2, 2019.

*Corresponding author: axb779@psu.edu
$1,567 \pm 2,165 \mathrm{ng} / \mathrm{mL}$, respectively). The correlation between Hp-MMP 9 in serum and VD was 0.22 ; nevertheless, it was not statistically significant. Interestingly, Hp and Hp-MMP 9 concentrations were higher in VD samples than in serum, regardless of VDS. Results from this study suggest that inflammatory biomarkers may be increased in cows with a VDS of 4 or 5 . Further research should be aimed at elucidating the processes involved in inflammatory biomarker production and transportation in the uterus, as well as the effect of these biomarkers on endometrial cells.

Key words: haptoglobin, matrix metalloproteinase-9, vaginal discharge, metritis

\section{Short Communication}

Clinical metritis is the infection of the layers of the uterus, characterized by the presence of an enlarged uterus and abnormal red-brownish foul-smelling vaginal discharge (VD), with or without the presence of systemic signs such as fever, anorexia, or depression (Barragan et al., 2018). It is a prevalent condition observed in dairy farms, and can affect 15 to $20 \%$ of cows (Gilbert, 2016). Clinical metritis causes important economic losses to dairy operations, because it decreases milk yield (Rajala and Gröhn, 1998), delays time to conception (Fourichon et al., 2000), and increases culling rates (Gröhn et al., 2003). It also negatively affects the well-being of dairy cows. It has been reported that dairy cows with clinical metritis had a higher back arch during uterine palpation through the rectum than cows without clinical metritis, suggesting that cows with clinical metritis may experience visceral pain (Stojkov et al., 2015). More recently, Barragan et al. (2018) reported that cows with clinical metritis at $7 \pm 3$ DIM had higher circulating concentrations of substance $\mathrm{P}$, a neuropeptide involved in pain transmission and inflammation (DeVane, 2001), and haptoglobin (Hp), an acute-phase protein secreted by the liver in response 
to cytokines (Cray et al., 2009), suggesting that clinical metritis cows experience not only inflammation but also pain.

Several authors (Drillich et al., 2007; Chan et al., 2010; Pohl et al., 2015) have shown that cows with clinical metritis had higher concentrations of $\mathrm{Hp}$ than cows without clinical metritis, and some (Huzzey et al., 2009) have suggested that circulating concentration of Hp before and after calving could be used as a predictor of clinical metritis. Other blood biomarkers, such as neutrophil-derived haptoglobin-matrix metalloproteinase 9 (Hp-MMP 9) complexes have been used to assess neutrophil activation and degranulation in inflammatory conditions (Bannikov et al., 2011; Hanthorn et al., 2014). For instance, Hanthorn et al. (2014) reported that serum Hp-MMP 9 was increased in calves inoculated with Bibersteinia trehalosi and Mannheimia haemolytica, concluding that Hp-MMP 9 could be used as an indicator of early pulmonary inflammation.

Screening the VD of postpartum dairy cows is a common practice to determine not only the presence of clinical metritis, but also its severity, which can be subjectively measured by assessing the color, proportion of pus, and odor of the VD (Sheldon et al., 2006). Others have reported that inflammatory mediators such as interleukins (IL-1 $\beta$, IL-6, and IL-8) and serum amyloid $\mathrm{A}$ in the vaginal mucus were increased in cows diagnosed with clinical endometritis $21 \mathrm{~d}$ after calving and concluded that VD was a convenient sample for profiling local inflammatory changes associated with uterine disease (Adnane et al., 2017). However, there is still a lack of knowledge regarding the usefulness of measuring these biomarkers in VD to objectively assess the severity of clinical metritis and the correlation between local uterine inflammation and systemic inflammation.

The objectives of this study were to assess (1) the concentrations of $\mathrm{Hp}$ and Hp-MMP 9 in the VD of postpartum cows, and (2) the correlation between serum and VD Hp and Hp-MMP 9 concentrations. We also assessed BHB concentrations and BCS for a more comprehensive picture of the effects of clinical metritis in dairy cattle. We hypothesized that Hp and Hp-MMP 9 concentrations would differ between cows with different VD scores, and that serum concentrations of $\mathrm{Hp}$ and Hp-MMP 9 would correlate with VD concentrations.

Fifty-seven dairy cows from 4 dairy farms in central Pennsylvania were enrolled in this observational study. Farm sizes ranged from 601 to 881 milking cows. Farm A milked approximately 601 cows and had a yearly rolling herd average milk yield of 10,527 kg; farm B milked approximately 749 cows and had a yearly rolling herd average milk yield of $12,765 \mathrm{~kg}$; farm C milked approxi- mately 850 cows and had a yearly rolling herd average milk yield of 11,482 kg; and farm D milked approximately 881 cows and had a yearly rolling herd average milk yield of 13,182 kg. All farms housed animals in sand bedded, 6-row freestall barns. A TMR formulated to meet or exceed dietary nutritional requirements for postpartum dairy cows (NRC, 2001) was delivered twice daily. Feed push-ups were performed approximately every 2 to $4 \mathrm{~h}$. Postpartum cows were milked 3 times daily. The procedures described below were reviewed and approved by the Institutional Animal Care Use Committee at Pennsylvania State University (protocol number: PRAMS200545723).

Each participant farm was visited once by the study team. We obtained a list of animals from on-farm computer records to select the study subjects. Every postpartum cow in the desired DIM range $(7 \pm 3$ DIM; $\mathrm{n}=57$ ) was screened for clinical metritis using a Metricheck device (Simcro Tech Ltd., Hamilton, New Zealand). Postpartum cows that had a health event (milk fever, $\mathrm{n}=1$; mastitis, $\mathrm{n}=1$ ) before study enrollment or showed any signs of other disease (pneumonia, $\mathrm{n}=$ 1 ; lameness, $\mathrm{n}=1$ ) at enrollment were excluded from the study. Therefore, a total of 53 cows remained in the study. We scored VD using a 5-point scale, adapted from Williams et al. (2005), to categorize the severity of clinical metritis. Briefly, VD score (VDS) was assessed by one trained veterinarian based on color, proportion of pus, and odor: 1 , clear fluid $(\mathrm{n}=4) ; 2,<50 \%$ white purulent fluid $(\mathrm{n}=14) ; 3,>50 \%$ white purulent fluid $(\mathrm{n}=8) ; 4$, red-brownish watery fluid without fetid smell $(\mathrm{n}=22)$; and 5, fetid red-brownish watery fluid $(\mathrm{n}=5)$. Cows with a VDS of 5 were classified as having clinical metritis. Samples of VD were collected using the Metricheck device to assess Hp and Hp-MMP 9. Samples were collected in 50-mL polypropylene conical tubes (Becton Dickinson and Co., Franklin Lakes, $\mathrm{NJ}$ ) and placed in a cooler with ice immediately after collection. Samples were stored at $-20^{\circ} \mathrm{C}$, within $2 \mathrm{~h}$ of collection, until analysis.

We also collected blood samples from the coccygeal blood vessels into 8.5-mL evacuated serum separator tubes (Vacutainer; Becton, Dickinson and Co.) to determine serum concentrations of $\mathrm{BHB}, \mathrm{Hp}$, and $\mathrm{Hp}-$ MMP 9. Blood samples were stored on ice immediately after collection and centrifuged within $2 \mathrm{~h}$ of collection. Serum harvested from the blood samples was stored at $-20^{\circ} \mathrm{C}$ until analysis. In addition, BCS was assessed at study enrollment using a 5-point scale (Ferguson et al., 1994).

Serum samples were analyzed using an electronic handheld device (PortaCheck, Moorestown, NJ) to determine BHB concentrations. An animal with a se- 
rum $\mathrm{BHB}$ concentration $>1.2 \mathrm{mmol} / \mathrm{L}$ was considered to have ketosis (Iwersen et al., 2009). We determined serum and VD concentrations of Hp using a commercially available bovine haptoglobin ELISA kit (Life Diagnostics, West Chester, PA) following the manufacturer's instructions. Frozen VD samples were thawed at room temperature. Each sample was then centrifuged at $3,000 \times g$ in a precooled centrifuge $\left(4^{\circ} \mathrm{C}\right)$ for 30 min, and vortexed for $30 \mathrm{~s}$ to mix fluid. Samples $(20$ $\mu \mathrm{L}$ ) were diluted 1:2,000 (Hp) and 1:40 (Hp-MMP 9). Samples with an optical density greater than the high standard of the calibration curve were subsequently rediluted within the range of the $\mathrm{Hp}$ (250 to $3.9 \mathrm{ng}$ / $\mathrm{mL}$ ) or Hp-MMP 9 (174 to $2.7 \mathrm{ng} / \mathrm{mL}$ ). After the optical density was within the range of the standard curve, the concentration of the analyte in each sample was corrected for dilution.

To evaluate VD and serum Hp, affinity-purified bovine $\mathrm{Hp}$ was diluted to create a standard curve (250 ng/ $\mathrm{mL}$ to $3.9 \mathrm{ng} / \mathrm{mL}$ ) using sample buffer. We used the analyte optical densities and their corresponding expected concentrations to calculate the observed concentration using a 4-parameter logistic regression analysis. The analyte recovery from these calibrators was $98 \pm 2 \%$. The slope of the lines $(0.99 \pm 0.03)$ and the correlation coefficient $(0.997 \pm 0.0023)$ of the calibration curves were considered acceptable. The average coefficients of variation for $\mathrm{VD}$ and serum sample $\mathrm{Hp}$ within and between plates were 4.9 and $5.2 \%$, respectively.

The serum and VD concentrations of Hp-MMP 9 were determined using an in-house ELISA assay as previously described (Bannikov et al., 2007). To validate VD Hp-MMP 9, control serum containing 1,392 ng/mL of Hp-MMP 9 was serially diluted using sample buffer from $1: 2(696 \mathrm{ng} / \mathrm{mL})$ to $1: 2,048(0.679 \mathrm{ng} / \mathrm{mL})$ on 4 separate occasions. Analyte optical densities were determined as described for haptoglobin, and the concentrations determined by 4-parameter logistic regression analysis. The analyte recovery was $101 \pm 2.5 \%$. The average slope $(1.01 \pm 0.04)$ and coefficient of correlation $(0.997 \pm 0.002)$ of these analyses were considered acceptable. The average coefficient of variation for VD and serum Hp-MMP 9 samples within and between plates were 3.2 and $4.3 \%$, respectively. The lower limits of quantitation of the Hp and Hp-MMP 9 assays were $3.9 \mathrm{ng} / \mathrm{mL}$ and $2.7 \mathrm{ng} / \mathrm{mL}$, respectively.

Statistical analyses of this observational cross-sectional study were performed using SAS software, version 9.4 (SAS Institute Inc., Cary, NC). Cow was used as the experimental unit. The normality and homogeneity of variances of the dependent variables were assessed through graphical methods (histogram and Q-Q plot), Shapiro-Wilk statistics, and Bartlett's tests using the
UNIVARIATE procedure in SAS. Due to lack of normality, VD Hp, VD Hp-MMP 9, and serum Hp-MMP 9 were $\log _{10}$-transformed for analysis. We have reported the geometric means and $95 \%$ confident intervals of the back-transformed results in this study. Continuous dependent variables (i.e., VD Hp, VD Hp-MMP 9, serum Hp-MMP 9, BCS, BHB) were analyzed by using a mixed linear regression model with the PROC MIXED procedure in SAS. The variables initially offered to the model were farm, parity, DIM, BCS, ketosis, and VDS. Using the Wald statistic backward selection criterion $(P>0.15)$ we removed nonsignificant variables. Cow within farm was included in the model as random effect. For VD Hp, the variables that remained in the final model were VDS, DIM, ketosis, and BCS. For VD Hp-MMP 9, farm was left in the model because of its statistical significance $(P=0.03)$; therefore, only the cow variable was included in the random effect. The variables that remained in the final model for VD HpMMP 9 were VDS, DIM, ketosis, and farm. For serum Hp-MMP 9, farm was left in the model because of its statistical significance $(P=0.006)$; therefore, only the cow variable was included in the random effect. The variables that remained in the final model for serum Hp-MMP 9 were clinical metritis and farm. Because of the failure of serum Hp to achieve normality after data transformation $\left(\log _{10}, \mathrm{R}^{2}\right)$, we analyzed it using the nonparametric Kruskal-Wallis test using the NPAR1WAY procedure in SAS. Mean and standard deviation values are reported. The proportion of animals with ketosis based on the concentration of BHB between groups were analyzed by using multivariable logistic regression using the GLIMMIX procedure in SAS. The variables of interest, as well as their interactions, were considered significant if $P<0.05 ; P<0.10$ was considered a tendency. Pearson correlation coefficients were computed using the PROC CORR procedure in SAS for the correlation analysis between Hp and Hp-MMP 9 concentrations in serum and VD. The variables VD Hp, VD Hp-MMP 9, serum Hp, and serum Hp-MMP 9 were $\log 10$-transformed before correlation analysis.

We found no difference between study groups in BCS or subclinical ketosis incidence. Similar results were reported by Barragan et al. (2018), where the authors described that the incidence of subclinical ketosis was not different between cows diagnosed with or without clinical metritis at 7 DIM. However, in the same study, the authors reported that cows with clinical metritis had a lower BCS than cows without clinical metritis. The smaller sample size and less homogeneous study groups in the present study compared with the latter study (cows with and without clinical metritis were matched based on DIM and lactation) may explain, at 


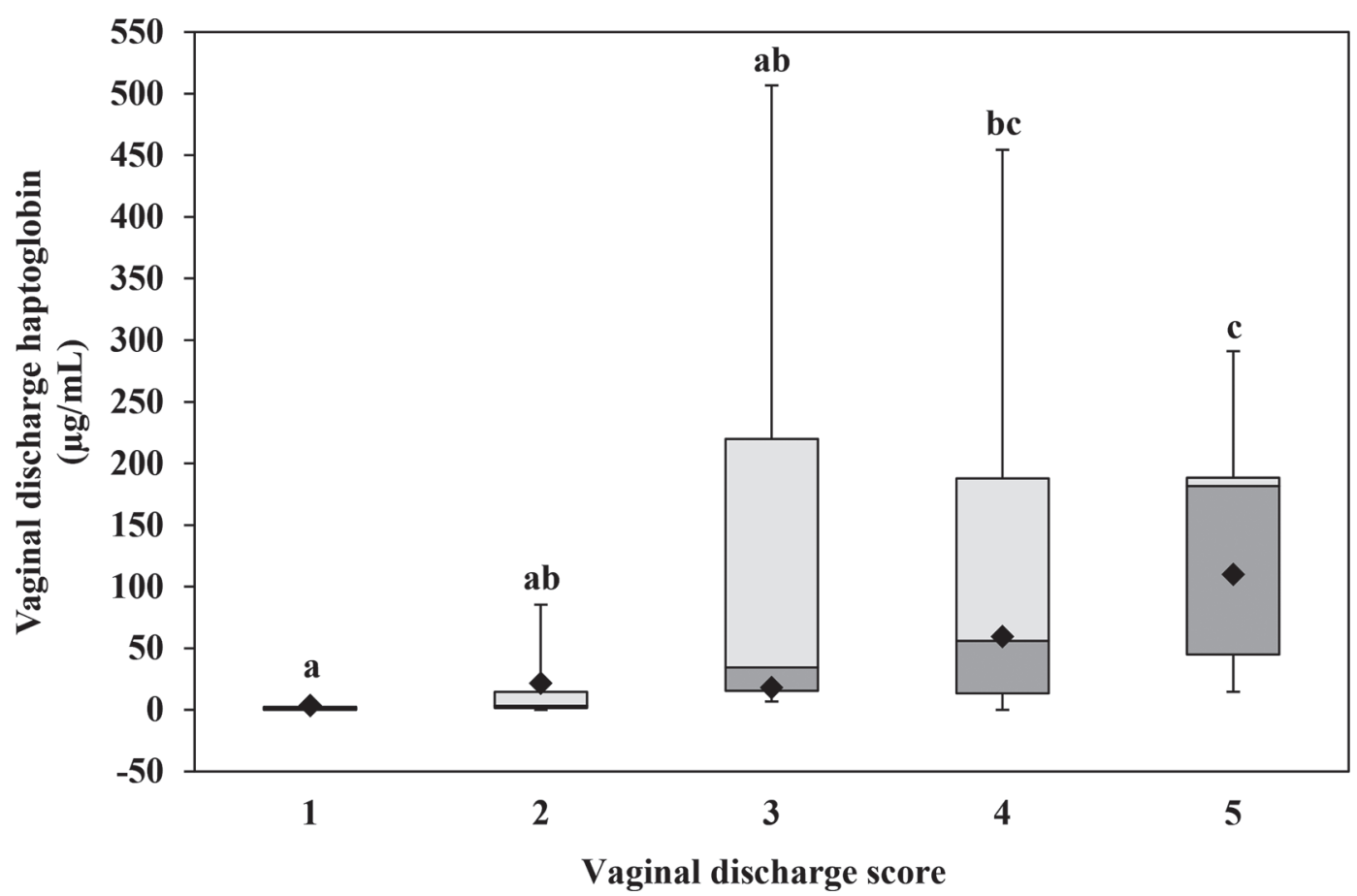

Figure 1. Concentration of haptoglobin $(\mu \mathrm{g} / \mathrm{mL})$ by vaginal discharge scores: 1 , clear fluid $(\mathrm{n}=4) ; 2,<50 \%$ white purulent fluid $(\mathrm{n}=14)$; $3,>50 \%$ white purulent fluid $(\mathrm{n}=8) ; 4$, red-brownish watery fluid without fetid smell $(\mathrm{n}=22) ; 5$, fetid red-brownish watery fluid $(\mathrm{n}=5)$. Box plots depict first quartile (25th percentile, dark gray), median (solid line), and third quartile (75th percentile, light gray), and the whiskers indicate minimum and maximum values from the raw data. Solid diamonds represent the back-transformed LSM obtained from the statistical model. Different letters $(\mathrm{a}, \mathrm{b}, \mathrm{c})$ indicate statistical significance at $P<0.05$.

least in part, why the present study failed to observe differences in BCS between cows with and without clinical metritis.

Because there was no statistically significant difference in $\mathrm{Hp}$ concentrations between cows with VDS of 4 and 5, we grouped these cows for analysis (VDS4/5). Similarly, cows with VDS of 1,2 , or 3 were combined into a single group (VDS1/3). Results are presented comparing these 2 groups. However, the small sample size of our study may not have allowed us to convincingly prove a lack of difference in $\mathrm{Hp}$ concentrations between cows with VDS of 4 or 5 ; further research with a larger sample size is needed to confirm this assumption.

Cows with VDS4 $/ 5$ had a higher $(P<0.0001)$ concentration of serum Hp than cows with VDS1/3 (93 \pm $187 \mu \mathrm{g} / \mathrm{mL}$ vs. $59 \pm 106 \mu \mathrm{g} / \mathrm{mL}$, respectively). These results agreed with those of previous studies (Drillich et al., 2007; Chan et al., 2010; Pohl et al., 2015), but also suggested that cows with a VDS of 4 presented with a systemic inflammatory response similar to cows with classic metritis (i.e., VDS 5). For instance, Huzzey et al. (2009) showed that cows with elevated Hp concentrations $(>1 \mathrm{~g} / \mathrm{L})$ at $3 \mathrm{~d}$ after calving were 6.7 times more likely to develop clinical metritis. Similarly, Bar- ragan et al. (2018) reported that cows diagnosed with clinical metritis at 7 DIM had higher concentrations of Hp. Increased concentrations of Hp have been also associated with other common postpartum diseases, such as mastitis and low fertility in dairy cows (Grönlund et al., 2005; Dubuc et al., 2010; Huzzey et al., 2015). Although our results for serum Hp agreed with previous studies, the $\mathrm{Hp}$ concentrations of the cows in our study were lower. The severity of clinical metritis can affect $\mathrm{Hp}$ concentrations, especially if the animals are systemically ill. In our study, enrolled animals were not severely affected systemically by CM, as they were neither off feed nor had obvious signs of systemic illness (e.g., depression) upon enrollment. In the previous studies, more animals could have been systemically ill. Furthermore, the days after calving at which $\mathrm{Hp}$ was measured could have affected overall Hp concentrations in the study animals. Physiologically, Hp concentration peaks at calving and decreases 1 week after (Uchida et al., 1993). All of this may explain, at least in part, the difference in overall $\mathrm{Hp}$ concentrations between the animals in our study and those in previous studies.

Cows with VDS4/5 had higher $(P=0.007)$ concentration of VD Hp than cows with VDS1/3 $(73 \pm 56 \mu \mathrm{g} /$ $\mathrm{mL}$ vs. $17 \pm 16 \mu \mathrm{g} / \mathrm{mL}$, respectively). Analysis by VDS 
showed that VDS 4 and 5 had the highest Hp concentrations compared with other scores (VDS $1=3 \pm 17$ $\mu \mathrm{g} / \mathrm{mL} ; \operatorname{VDS} 2=21 \pm 29 \mu \mathrm{g} / \mathrm{mL} ; \operatorname{VDS} 3=18 \pm 30$ $\mu \mathrm{g} / \mathrm{mL} ; \operatorname{VDS} 4=59 \pm 48 \mu \mathrm{g} / \mathrm{mL} ; \operatorname{VDS} 5=109 \pm 201$ $\mu \mathrm{g} / \mathrm{mL}$; Figure 1). Furthermore, we found a significant correlation $(0.37 ; P=0.013$; Figure 2$)$ between the levels of Hp in serum and in VD. Because Hp is produced by the liver in response to a systemic inflammatory event and is considered a biomarker of systemic inflammation (Cray et al., 2009), the concentration of $\mathrm{Hp}$ in the local inflammation site would be expected to be low. However, in the present study the concentration of Hp in VD was higher $(P=0.01)$ than the concentration in serum $(25 \pm 15 \mu \mathrm{g} / \mathrm{mL}$ vs. $10 \pm 6 \mu \mathrm{g} / \mathrm{mL}$, respectively), regardless of VDS. These results suggest that Hp may diffuse from the blood stream to the inflammation site, and this may alter the normal functioning of endometrial cells. An alternative to this speculation is that neutrophil influx provided additional $\mathrm{Hp}$ in the uterine lumen or that local (endometrial) production of Hp occurs in the cow (Cooray et al., 2007; SharpeTimms et al., 2010; Adnane et al., 2017).

The VD Hp-MMP 9 concentration was higher $(P=$ $0.045)$ in VDS4/5 cows than in VDS1/3 cows $(7,629 \pm$ $9,847 \mathrm{ng} / \mathrm{mL}$ vs. $1,567 \pm 2,165 \mathrm{ng} / \mathrm{mL}$, respectively).
We observed a numerical difference in serum Hp-MMP 9 concentrations between VDS4/5 and VDS1/3 cows $(49 \pm 36 \mathrm{ng} / \mathrm{mL}$ vs. $38 \pm 33 \mathrm{ng} / \mathrm{mL}$, respectively), but this difference was not statistically significant $(P$ $=0.61$ ). We speculate that systemic release of the neutrophil granule Hp-MMP 9 was not occurring in either group, but this finding may also have been the result of the small sample size in this study. The correlation between Hp-MMP 9 in serum and VD was 0.22 , but this correlation was not statistically significant $(P=0.19$; Figure 3). Moreover, regardless of VDS, the concentration of Hp-MMP 9 in VD was higher $(P<0.0001)$ than in serum $(3,061 \pm 2,175 \mathrm{ng} / \mathrm{mL}$ vs. $52 \pm 43 \mathrm{ng} /$ $\mathrm{mL}$, respectively). Because Hp-MMP 9 is an indicator of neutrophil activation and degranulation (Bannikov et al., 2011), this parameter would be expected to be higher in the VD of postpartum cows, where neutrophils may be actively recruited to eliminate bacteria, cellular debris, and other debris.

The results of this study suggest that $\mathrm{Hp}$ and $\mathrm{Hp}$ MMP 9 are increased in cows with VDS of 4 and 5; however, further research should be aimed at elucidating the mechanisms involved in the uterine local production or transportation into the uterus of Hp and HpMMP 9, while evaluating the potential negative effects

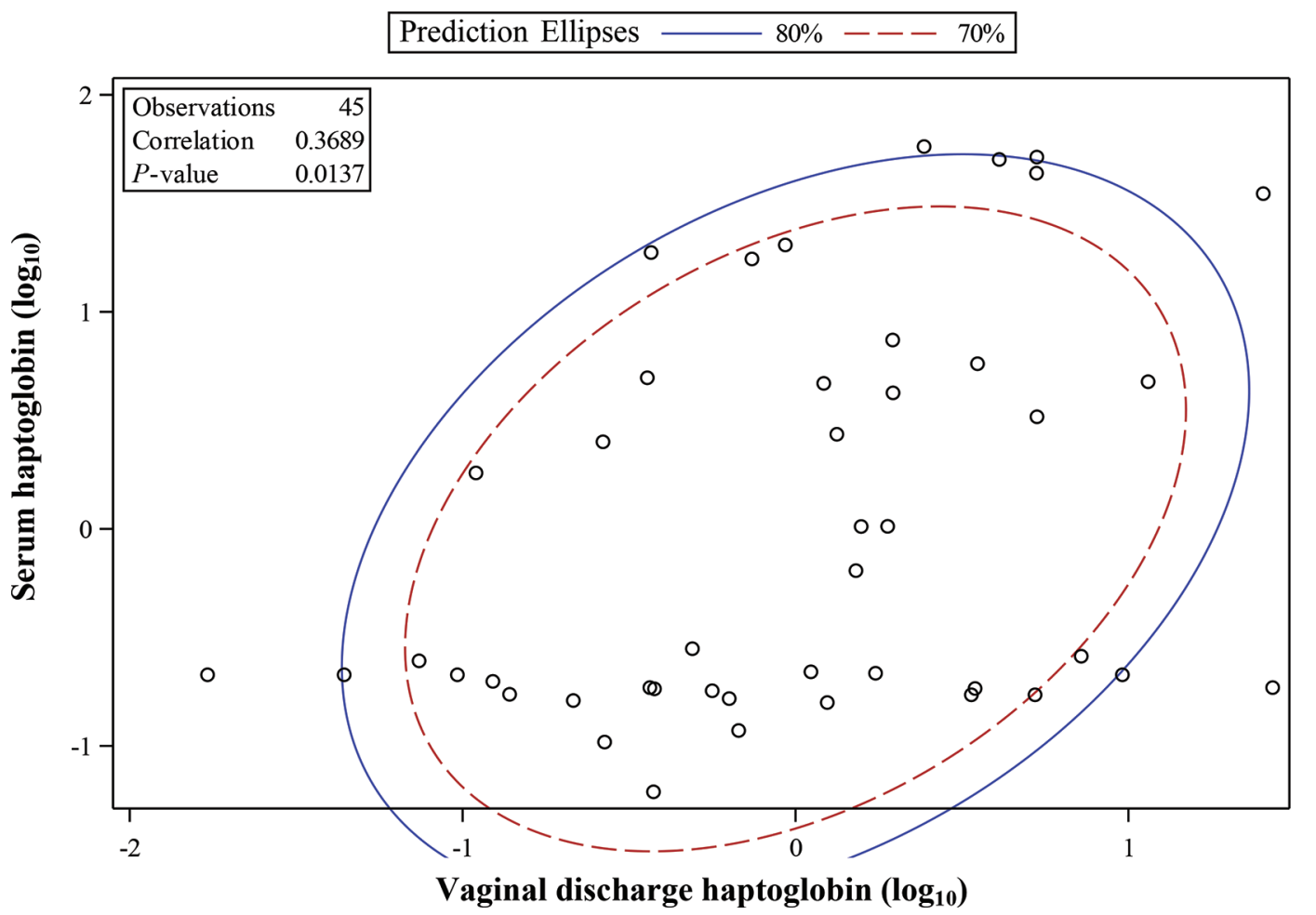

Figure 2. Scatter plot showing the correlation between the concentration of serum haptoglobin $\left(\log _{10}\right.$-transformed) and vaginal discharge of study cows. The prediction ellipses approximate a region that contains a specified percentage of the population (70 and $80 \%)$ and are centered at the means. 


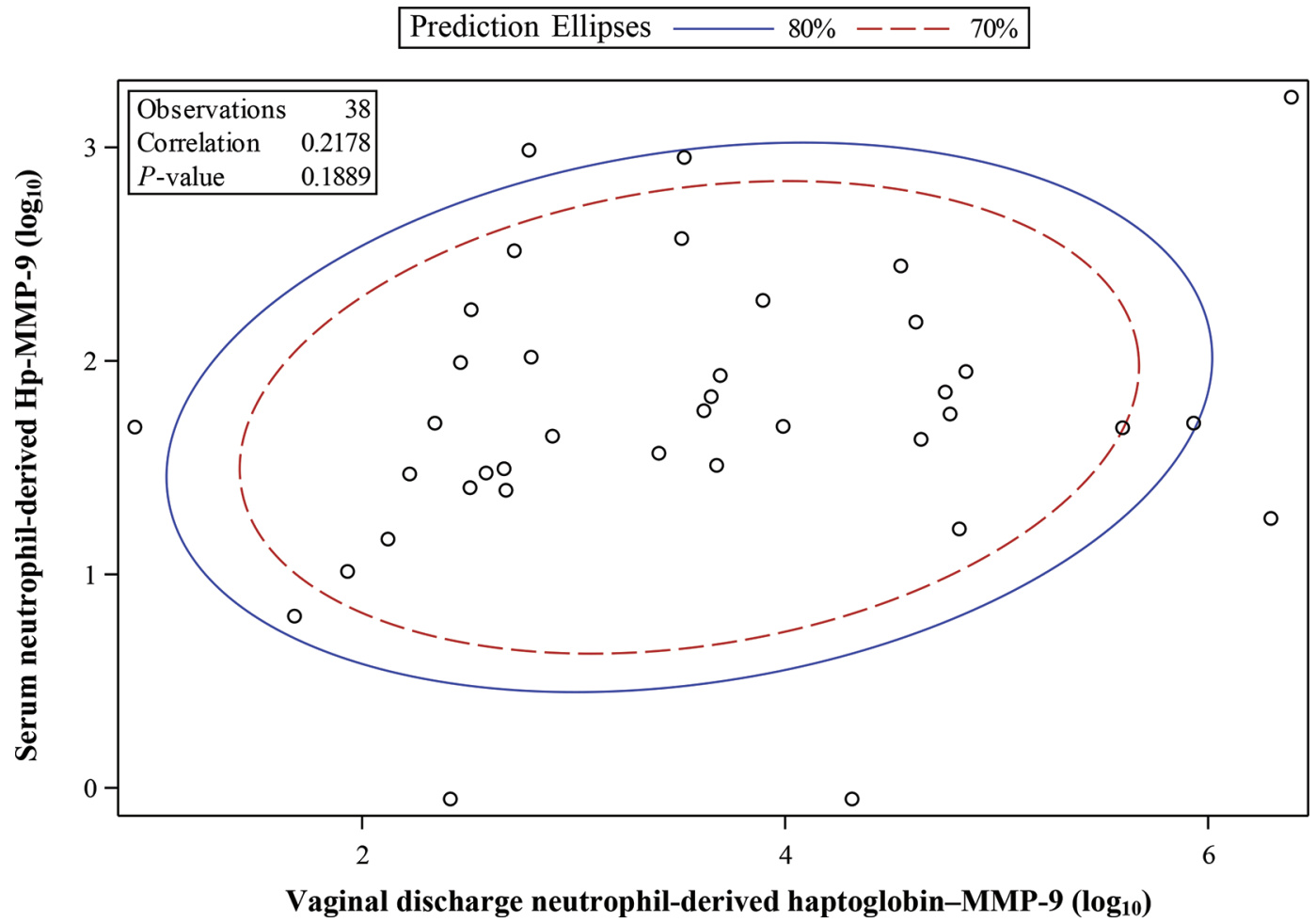

Figure 3. Scatter plot showing the correlation between the concentration of serum neutrophil-derived haptoglobin-matrix metalloproteinase 9 (Hp-MMP-9; $\log _{10}$-transformed) and the vaginal discharge of study cows. The prediction ellipses approximate a region that contains a specified percentage of the population (70 and $80 \%$ ) and are centered at the means.

of these inflammatory biomarkers on endometrial cell function and assessing the effects of anti-inflammatory therapy on the concentration of these molecules in the VD of postpartum dairy cows.

\section{ACKNOWLEDGMENTS}

The authors thank the farms that permitted us to collect samples from their animals for this study, as well as the undergraduate students who assisted in field data collection and data uploading. Special thanks to Louise Byler, Rebecka Russel, and Dare Sheperd (Department of Veterinary and Biomedical Sciences, Pennsylvania State University, University Park) for their contribution to this study. The authors also acknowledge the support provided by the Vernon L. Tharp Professorship to J. Lakritz and by the USDA National Institute of Food and Agriculture and Federal Appropriations under Project PEN04665 and Accession Number 1017918.

\section{REFERENCES}

Adnane, M., A. Chapwanya, R. Kaidi, K. G. Meade, and C. O'Farrelly. 2017. Profiling inflammatory biomarkers in cervico-vaginal mucus (CVM) postpartum: Potential early indicators of bovine clinical endometritis? Theriogenology 103:117-122.
Bannikov, G. A., C. A. Hinds, P. J. Rajala-Schultz, C. Premanandan, D. M. Rings, and J. Lakritz. 2011. Serum haptoglobin-matrix metalloproteinase 9 (Hp-MMP 9) complex as a biomarker of systemic inflammation in cattle. Vet. Immunol. Immunopathol. 139:41-49.

Bannikov, G. A., J. S. Mattoon, E. J. Abrahamsen, C. Premanandan, K. B. Green-Church, A. E. Marsh, and J. Lakritz. 2007. Biochemical and enzymatic characterization of purified covalent complexes of matrix metalloproteinase- 9 and haptoglobin released by bovine granulocytes in vitro. Am. J. Vet. Res. 68:995-1004.

Barragan, A. A., J. M. Piñeiro, G. M. Schuenemann, P. J. RajalaSchultz, D. E. Sanders, J. Lakritz, and S. Bas. 2018. Assessment of daily activity patterns and biomarkers of pain, inflammation, and stress in lactating dairy cows diagnosed with clinical metritis. J. Dairy Sci. 101:8248-8258.

Chan, J. P.-W., C.-C. Chang, W.-L. Hsu, W.-B. Liu, and T.-H. Chen. 2010. Association of increased serum acute-phase protein concentrations with reproductive performance in dairy cows with postpartum metritis. Vet. Clin. Pathol. 39:72-78.

Cooray, R., K. P. Waller, and P. Venge. 2007. Haptoglobin comprises about $10 \%$ of granule protein extracted from bovine granulocytes isolated from healthy cattle. Vet. Immunol. Immunopathol. 119:310-315.

Cray, C., J. Zaias, and N. H. Altman. 2009. Acute phase response in animals: A review. Comp. Med. 59:517-526.

DeVane, C. L. 2001. Substance P: A new era, a new role. Pharmacotherapy 21:1061-1069.

Drillich, M., D. Voigt, D. Forderung, and W. Heuwieser. 2007. Treatment of acute puerperal metritis with flunixin meglumine in addition to antibiotic treatment. J. Dairy Sci. 90:3758-3763.

Dubuc, J., T. F. Duffield, K. E. Leslie, J. S. Walton, and S. J. LeBlanc. 2010. Risk factors for postpartum uterine diseases in dairy cows. J. Dairy Sci. 93:5764-5771. 
Ferguson, J. D., D. T. Galligan, and N. Thomsen. 1994. Principal descriptors of body condition score in Holstein cows. J. Dairy Sci. $77: 2695-2703$.

Fourichon, C., H. Seegers, and X. Malher. 2000. Effect of disease on reproduction in the dairy cow: A meta-analysis. Theriogenology 53:1729-1759.

Gilbert, R. O. 2016. Management of reproductive disease in dairy cows. Vet. Clin. North Am. Food Anim. Pract. 32:387-410.

Gröhn, Y. T., P. J. Rajala-Schultz, H. G. Allore, M. A. DeLorenzo, J. A. Hertl, and D. T. Galligan. 2003. Optimizing replacement of dairy cows: Modeling the effects of diseases. Prev. Vet. Med. $61: 27-43$

Grönlund, U., C. H. Sandgren, and K. P. Waller. 2005. Haptoglobin and serum amyloid A in milk from dairy cows with chronic subclinical mastitis. Vet. Res. 36:191-198.

Hanthorn, C. J., G. A. Dewell, R. D. Dewell, V. L. Cooper, C. Wang, P. J. Plummer, and J. Lakritz. 2014. Serum concentrations of haptoglobin and haptoglobin-matrix metalloproteinase 9 (Hp-MMP 9) complexes of bovine calves in a bacterial respiratory challenge model. BMC Vet. Res. 10:285.

Huzzey, J. M., T. F. Duffield, S. J. LeBlanc, D. M. Veira, D. M. Weary, and M. A. G. Von Keyserlingk. 2009. Haptoglobin as an early indicator of metritis. J. Dairy Sci. 92:621-625.

Huzzey, J. M., S. Mann, D. V. Nydam, R. J. Grant, and T. R. Overton. 2015. Associations of peripartum markers of stress and inflammation with milk yield and reproductive performance in Holstein dairy cows. Prev. Vet. Med. 120:291-297.

Iwersen, M., U. Falkenberg, R. Voigtsberger, D. Forderung, and W. Heuwieser. 2009. Evaluation of an electronic cowside test to detect subclinical ketosis in dairy cows. J. Dairy Sci. 92:2618-2624.
National Research Council. 2001. Nutrient Requirements of Dairy Cattle. 7th rev. ed. Natl. Acad. Press, Washington, DC

Pohl, A., O. Burfeind, and W. Heuwieser. 2015. The associations between postpartum serum haptoglobin concentration and metabolic status, calving difficulties, retained fetal membranes, and metritis. J. Dairy Sci. 98:4544-4551.

Rajala, P. J., and Y. T. Gröhn. 1998. Effects of dystocia, retained placenta, and metritis on milk yield in dairy cows. J. Dairy Sci. $81: 3172-3181$.

Sharpe-Timms, K. L., H. Nabli, R. L. Zimmer, J. A. Birt, and J. W. Davis. 2010. Inflammatory cytokines differentially up-regulate human endometrial haptoglobin production in women with endometriosis. Hum. Reprod. 25:1241-1250.

Sheldon, I. M., G. S. Lewis, S. LeBlanc, and R. O. Gilbert. 2006. Defining postpartum uterine disease in cattle. Theriogenology 65:1516-1530.

Stojkov, J., M. A. G. von Keyserlingk, J. N. Marchant-Forde, and D. M. Weary. 2015. Assessment of visceral pain associated with metritis in dairy cows. J. Dairy Sci. 98:5352-5361.

Uchida, E., N. Katoh, and K. Takahashi. 1993. Appearance of haptoglobin in serum from cows at parturition. J. Vet. Med. Sci. $55: 893-894$.

Williams, E. J., D. P. Fischer, D. U. Pfeiffer, G. C. England, D. E. Noakes, H. Dobson, and I. M. Sheldon. 2005. Clinical evaluation of postpartum vaginal mucus reflects uterine bacterial infection and the immune response in cattle. Theriogenology 63:102-117. 